\title{
Zhi Zhen Fang formula reverses Hedgehog pathway mediated multidrug resistance in colorectal cancer
}

\author{
HUA SUI ${ }^{1,2^{*}}$, PEIWEN DUAN ${ }^{1 *}$, PIAOTING GUO $^{3}$, LISHUANG HAO $^{4}$, XUAN LIU $^{5}$, JINHUA ZHANG $^{2}$, \\ HUIRONG ZHU ${ }^{2}$, MIAOMIAO ZHAO ${ }^{1}, \mathrm{HAO} \mathrm{WANG}^{1}, \mathrm{QI} \mathrm{LI}^{2}$ and SONGPO WANG ${ }^{1}$ \\ ${ }^{1}$ Department of Traditional Chinese Medicine, Shanghai General Hospital, Shanghai Jiao Tong University School \\ of Medicine, Shanghai 200240; ${ }^{2}$ Department of Medical Oncology, Shuguang Hospital, Shanghai University of \\ Traditional Chinese Medicine, Shanghai 201203; ${ }^{3}$ Department of General Practice, The Second Affiliated Hospital \\ of Zhejiang Chinese Medical University, Hangzhou, Zhejiang 310053; ${ }^{4}$ Department of Gynecology, Shanghai TCM- \\ Integrated Hospital, Shanghai University of Traditional Chinese Medicine, Shanghai 200082; ${ }^{5}$ Department of Traditional \\ Chinese Medicine, Changzheng Hospital, Second Military Medical University, Shanghai 200003, P.R. China
}

Received January 12, 2017; Accepted June 28, 2017

DOI: $10.3892 /$ or.2017.5917

\begin{abstract}
Zhi-Zhen-Fang (ZZR), a Traditional Chinese Medicine (TCM) formula, has been clinically used in China to treat drug-resistant colorectal cancer (CRC) patients as an adjuvant. In this study, the efficacy of ZZR in suppressing multidrug resistance (MDR) on $\mathrm{CRC}$ was evaluated in vitro and in vivo. We observed that ZZR enhanced the sensitivity of chemotherapeutic drugs and induced apoptosis in a dose- and time-dependent mannner in CRC MDR cells. Interestingly, signaling of Hedgehog pathway, particularly Gli1, was also inhibited by ZZR. This effect of ZZR in reversing drug resistance and suppressing Gli1 was attenuated by a Hedgehog activator (SAG). Furthermore, ZZR inhibited MDR CRC tumor growth in a xenograft mouse model as well as downregulated Glil levels. This study provided the first direct evidence demonstrating ZZR can attenuate MDR by repressing Hedgehog signaling in human CRC.
\end{abstract}

\section{Introduction}

Multidrug resistance (MDR) is one of the primary problems to efficient cancer therapy (1). Cancer cells may employ several mechanisms of drug resistance such as apoptosis, autophagy, DNA repair, efflux transporters, and epigenetic regulation to acquire multidrug resistance $(2,3)$.

Correspondence to: Dr Songpo Wang, Department of Traditional Chinese Medicine, Shanghai General Hospital, Shanghai Jiao Tong University School of Medicine, 100 Haining Road, Hongkou, Shanghai 200240, P.R. China

E-mail: 13386259791@163.com

${ }^{*}$ Contributed equally

Key words: colorectal cancer, multidrug resistance, Hedgehog pathway, ZZR, in vitro, in vivo
In 1980s, Hedgehog (Hh) signal transduction pathway research made remarkable progress in fruit fly (4). The Hedgehog signaling pathway regulates cell proliferation, cell fate and patterning, stem and progenitor cell maintenance, as well as self-renewal and tissue repair $(5,6)$. It is now known that Hh signaling has two distinct facets of action mechanisms: the canonical and non-canonical pathways $(7,8)$. The canonical pathway components include ligands (sonic $\mathrm{Hh}$, Indian $\mathrm{Hh}$, and desert $\mathrm{Hh}$ ), patched receptors (PTCH1, PTCH2), signal transducer smoothened (SMO), and transcription factors (Gli1, Gli2, Gli3) (9). In brief, it is about binding of Hedgehog ligands secreted from one cell and the PTCH receptor on another cell. The non-canonical pathway initiates Hedgehog signaling, via Shh-mediated ERK activation, Wnt signaling results in expression and activation of Gli proteins, and the atypical mutual interaction of core Hh pathway components (10-12).

Traditional Chinese Medicines (TCMs) have been used as medicines, or health supplements in China and in East Asia for millennia. Traditional Chinese prescriptions and formulae, based on TCM principles, have been considered to target multiple pathways, and have been used to treat cancer, such as breast carcinoma (13), gastric cancer (14) and colorectal cancer (15). Similar efficiency as the chemotherapeutic drugs, traditional Chinese prescriptions and formulae are capable of effectively controling cancer progression, improving quality of life and prolonging survival times.

ZZR, a TCM empirical prescription developed in Shanghai General Hospital, which is a recipe derived from a classic TCM principle (nvigorating spleen and detoxification and opening collateral). Some studies illustrated that a Chinese formula, named JPJD, played an important role in liver cancer cure through upregulating expression levels of ABCC2 and downregulating levels of OATP1B2 (16). Additionally, our in vivo study demonstrated that intragastric administration of ZZR remarkedly inhibited P-gp expression levels and increased the sensitivity to chemotherapy drugs in CRC MDR cells. Also, the underlying mechanisms of the anti-metastatic effects associated with this formula remain unknown. 
Hh signaling pathway can be considered an actionable target for therapeutic intervention in CRC. The present study explored how Hh signaling pathway regulated $A B C$ transporter-mediated MDR and potential mechanisms of ZZR extract by evaluating antitumor effects of this formula both in vitro and in vivo.

\section{Materials and methods}

Preparation of the extracts for ZZR. ZZR extract was prepared according to our previously published method (17), including Huang-Qi (Radix Astragali), Nv-Zhen-Zi (Fructus Ligustri Lucidi), Yi-yi-ren (Semen Coicis), Shi-Jian-Chuan (Salvia Chinensis), Ye-Pu-Tao-Teng (Vitis quinquangularis Rehd), Teng-Li-Gen (Actinidia arguta), and Zhi-Xiang-Fu (Cyperus rotundus L.). In brief, the above mentioned 6 herbs were mixed at a ratio of 6:3:6:6:6:6:2 for a total dry weight of $175 \mathrm{~g}$. The herb concoction was immersed in $55 \%$ ethanol $(1: 10 \mathrm{w} / \mathrm{v})$ for $4 \mathrm{~h}$ and refluxed for $1.5 \mathrm{~h}$. After filtration, the residue was again refluxed with $55 \%$ ethanol $(1: 8, w / v)$ for $1 \mathrm{~h}$ and filtered. The two decoctions were dried by lyophilization to obtain the ZZR extract with a yield of dried powder of $24.4 \%$. Simultaneous quantification of five major active constituents in the extract was conducted by highperformance liquid chromatography (HPLC). The extract was stored at $4^{\circ} \mathrm{C}$, and its preparations were standardized, regulated and quality controlled according to the guidelines defined by Chinese State Food and Drug Administration (SFDA).

Cell culture and reagents. The human colorectal cancer HCT-116 and HCT-8 parental cell lines were purchased from the Shanghai Cell Collection (Shanghai, China). HCT-116/ L-OHP cell line and HCT-8/5-FU cell line were generated and maintained in our laboratory. Cells were maintained in RPMI-1640 containing 10\% FBS, penicillin (100 U/ml), and streptomycin $(100 \mathrm{mg} / \mathrm{ml})$ (Invitrogen, Carlsbad, CA, USA) at $37^{\circ} \mathrm{C}$ in a $5 \% \mathrm{CO}_{2}$ humidified atmosphere. HCT-116/L-OHP cells were routinely maintained in a medium containing 5,000 ng/ml oxaliplatin (L-OHP), and HCT-8/5-FU cells were in $1,000 \mathrm{ng} / \mathrm{ml}$ (5-FU). L-OHP was purchased from Shenzhen Main Luck Pharmaceuticals Co., Ltd. (Shenzhen, China), 5-FU was purchased from Shanghai Xudong Haipu Pharmaceutical Co., Ltd. (Shanghai, China). Monoclonal antibodies against Ptch1, Gli1, Gli2, and GAPDH were products of Cell Signaling Technology (Beverly, MA, USA). GANT61 and SAG were purchased from Selleck (Houston, TX, USA).

Cell viability asays. Cell proliferation assay was conducted using the cell count kit CCK-8 (Sigma), as previously described (17). Briefly, cells were seeded in 96 -well plates at $1 \times 10^{4}$ cells/well. When the cells reached $60 \%$ confluence, the medium was removed and replaced with fresh medium containing varying concentrations of ZZR with or without chemotherapy drugs (L-OHP and 5-FU) and incubated for $48 \mathrm{~h}$. The absorbance was read at $450 \mathrm{~nm}$ using a microplate enzyme-linked immunosorbent assay reader (Labsystems Dragon, Wellscan). All experiments were performed with 5 wells per experiment and repeated at least three times. $\mathrm{IC}_{10}, \mathrm{IC}_{20}, \mathrm{IC}_{50}$ (the half maximal inhibitory concentration) was estimated according to the following formula respectively: $\mathrm{IC}_{50}=\lg -1[\mathrm{Xm}-\mathrm{i}(\Sigma \mathrm{P}-0.5)]$;
$\mathrm{Xm}$, the maximum concentration; i, logarithm of the concentration; $\Sigma$ P, sum of growth inhibition rate. $\mathrm{IC}_{10}$ and $\mathrm{IC}_{20}$ was calculated according to SPSS statistical software.

Apoptosis assay. Cells were plated in 6 -well plates $\left(1 \times 10^{6}\right.$ cells/well) and after treatment were trypsinized and analyzed using an Annexin V-fluorescein isothiocyanate detection kit (eBioscience Inc., San Diego, CA, USA). Three concentrations of ZZR (obtained from the result of ZZR Cell Viability assays) and L-OHP $(10 \mu \mathrm{M})$ were added for $24 \mathrm{~h}$. The samples were immediately processed using flow cytometry to detect the relative amount of Annexin V-FITC-positive-PI-negative cells, as previously described (17).

Western blot analysis. Equal protein per lysate was resolved on Tris-glycine gel, transferred onto nitrocellulose membrane, and blocked for $1 \mathrm{~h}$ with 5\% non-fat dry milk. Membranes were incubated with desired primary antibody: Ptch1, Gli1, and Gli2 with 1:1,000 (Cell Signaling Technology, Inc., Danvers, MA, USA) overnight at $4^{\circ} \mathrm{C}$ and then with appropriate secondary antibody as previously reported (18). Equal loading was confirmed with GAPDH $(0.1 \mu \mathrm{g} / \mathrm{ml})$. Densitometric analysis was performed using the Scion Imaging software (Scion Corp.). GAPDH was used as a control for each sample.

Animal experiments. Male athymic nude mice (NCr-nu), 8-12 weeks old, were purchased from Sino-British SIPPR/BK Lab Animal Co., Ltd. (Shanghai, China, license no. SCXK 2010-0012), and maintained under specific-pathogen-free conditions. All animal protocols were approved by the Institutional Animal Use and Care Committee. All the experiments and animal care were approved by Shanghai Medical Experimental Animal Care Commission and in accordance with the Provision and General Recommendation of Chinese Experimental Animals Administration Legislation.

Mice were subcutaneously injected with $1.0 \times 10^{6}$ HCT-116/ L-OHP cells per animal. When the tumors reach an average size of $100 \mathrm{~mm}^{3}$, the mice were randomized into 5 groups ( $n=6$ per group) and received intragastric administration of vehicle control, L-OHP and amixture of ZZR and L-OHP. Briefly, L-OHP was given as an intraperitoneal injection every 2 days and the injection dosage $(5 \mathrm{mg} / \mathrm{kg}$ ) was according to half of the maximum tolerated dose (MTD) of oxaliplatin as previously described (19). ZZR was given every day at the doses of $13.27,26.54$ and $53.08 \mathrm{~g} / \mathrm{kg}$. In the clinical practice of Chinese herbal medicine, ZZR is usually prescribed at a daily dose of $175 \mathrm{~g}$ of herbal materials. When this human dose was converted into an animal dose (a person of $60 \mathrm{~kg}$, and a conversion factor of 9.1 between human and mouse), it was equivalent to the middle dose $(26.54 \mathrm{~g} / \mathrm{kg})$ used in this study.

The body weight of the animals and the two perpendicular diameters (A and $\mathrm{B}$ ) of tumor were recorded every 3 days and tumor volume $(\mathrm{V})$ was estimated according to the following formula (17): $V=\pi / 6 x[(A+B) / 2]^{3}$. The curve of tumor growth was drawn according to tumor volume and time of implantation. Six mice were sacrificed by decapitation in each group on 28th day after treatment.

Immunohistochemical analysis. Hydrated paraffin sections were incubated in a blocking solution (10\% donkey 


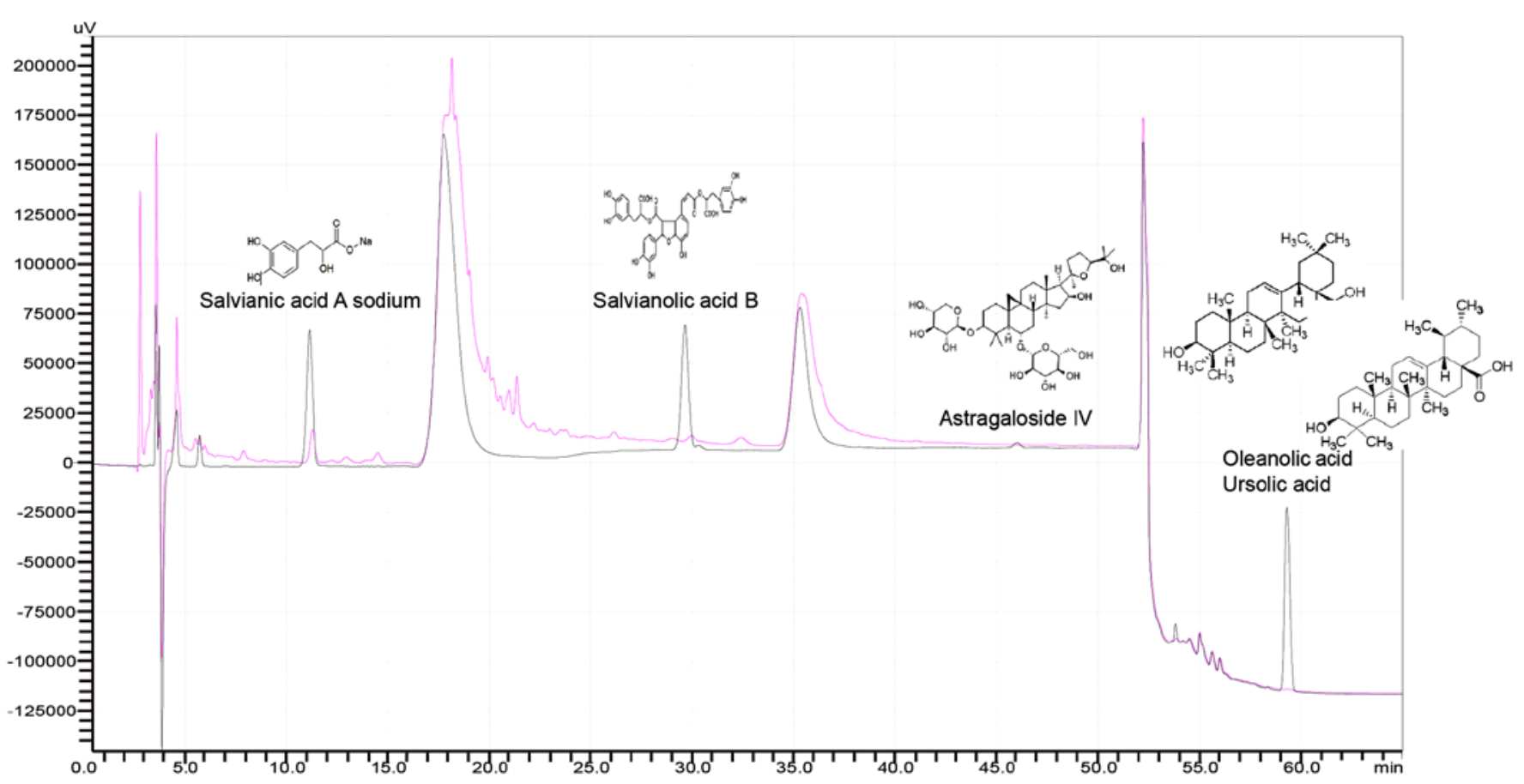

Figure 1. Base peak intensity chromatogram in negative ion modes for ZZR.

serum $+5 \%$ non-fat dry milk $+4 \% \mathrm{BSA}+0.1 \%$ Triton $\mathrm{X}-100$ ) for $10 \mathrm{~min}$, and then incubated at $4{ }^{\circ} \mathrm{C}$ overnight with antiGli1 (1:500). The analysis was conducted as described previously (19).

Statistical analysis. All quatitative data were expressed as mean \pm standard of at least three independent experiments. Statistical analyses were conducted using the Student's t-test. $\mathrm{P}<0.05$ or $\mathrm{P}<0.01$ were considered statistically significant.

\section{Results}

Analysis of active compounds in ZZR. To ensure the quality and stability of ZZR formula, we characterized the active components and their concentration using HPLC. As shown in Fig. 1, compared to standards, the amount of salvianic acid A sodium, salvianolic acid B and astragaloside IV are lower in ZZR ethanol extraction, but higher than that of ZZR water extract (data not shown). Thus, in this study, 55\% ethanol was adopted for ZZR extraction. As the ursolic acid and oleanolic acid isomers are inseparable (Fig. 1), salvianic acid A sodium, salvianolic acid B and astragaloside IV were therefore predicted to be the major antitumor components of ZZR solution.

Non-cytotoxic dose of ZZR. To rule out the possibility of cytotoxicity-induced inhibition of cell proliferation, we determined the cytotoxic effect of ZZR on colorectal cancer cells HCT-116 and its MDR derived cells HCT-116/L-OHP. Our data revealed: the doses of $\mathrm{IC}_{10}$ and $\mathrm{IC}_{20}$ in HCT-116 cells were 103.44 and $212.57 \mu \mathrm{g} / \mathrm{ml}, 122.64$ and $248.00 \mu \mathrm{g} / \mathrm{ml}$ in HCT-116/L-OHP cells, respectively (Fig. 2A). In any lower doses, little effect was observed on cell survival, thus for the following experiments, cells were treated with ZZR below the dose of $200 \mu \mathrm{g} / \mathrm{ml}$ which was roughly $\mathrm{IC}_{20}$.
ZZR enhances chemo-sensitivity of colorectal cancer cells. To determine if ZZR synergized with chemotherapeutic drugs in treating colorectal cancer cells, HCT-116 and HCT-116/L-OHP cells were treated with ZZR and L-OHP and 5-FU at $\mathrm{IC}_{50}$. As shown in Fig. 2B, ZZR induced significant cytotoxicity in HCT-116/L-OHP cells, decreasing L-OHP IC $\mathrm{I}_{50}$ from $111.03 \pm 20.60$ to $20.48 \pm 6.19 \mu \mathrm{M}$ and $16.23 \pm 5.31 \mu \mathrm{M}$ with 100 or $200 \mu \mathrm{g} / \mathrm{ml}$ of ZZR respectively, close to the $\mathrm{IC}_{50}$ in the parental cells which was $10.30 \pm 1.53 \mu \mathrm{M}$. Similarly, the $\mathrm{IC}_{50}$ of 5 -FU decreased from $165.03 \pm 17.97$ to $114.65 \pm 19.08 \mu \mathrm{M}$ and $95.03 \pm 9.22 \mu \mathrm{M}$ with 100 or $200 \mu \mathrm{g} / \mathrm{ml}$ of $\mathrm{ZZR}$ in a dose-dependent manner (Fig. 2C). These results suggested that ZZR had synergistic effect in combination with chemotherapy in treating human colorectal cancer.

ZZR induces apoptosis without disturbing cell cycle colorectal cancer cells. To explore the mechanisms of antiproliferative effect of ZZR, flow cytometry analysis was performed to determine if apoptosis was induced by L-OHP/ZZR combination. As shown in Fig. 3A, the percentages of apoptotic cells (including the early and late apoptotic cells) induced by $0,50,100$ and $200 \mu \mathrm{g} / \mathrm{ml}$ of ZZR were $11.41 \pm 0.57,16.01 \pm 0.92$, $17.45 \pm 1.03$ and $23.43 \pm 0.66 \%$, respectively. This indicates that ZZR augmented L-OHP induced cell apoptosis in a dose-dependent manner. However, the cell cycle analyses obtained from the flow cytometric analysis showed little impact of ZZR (Fig. 3B). These data suggested that the antiproliferative effect of ZZR was mostly via the direct induction of apoptosis.

Effects of ZZR on the Hedgehog pathway in the chemotherapeutic reagent-sensitive, and-insensitive colorectal cancer cells. To further elucidate the action mechanisms of ZZR in MDR colorectal cancer cells, we investigated if ZZR affected 

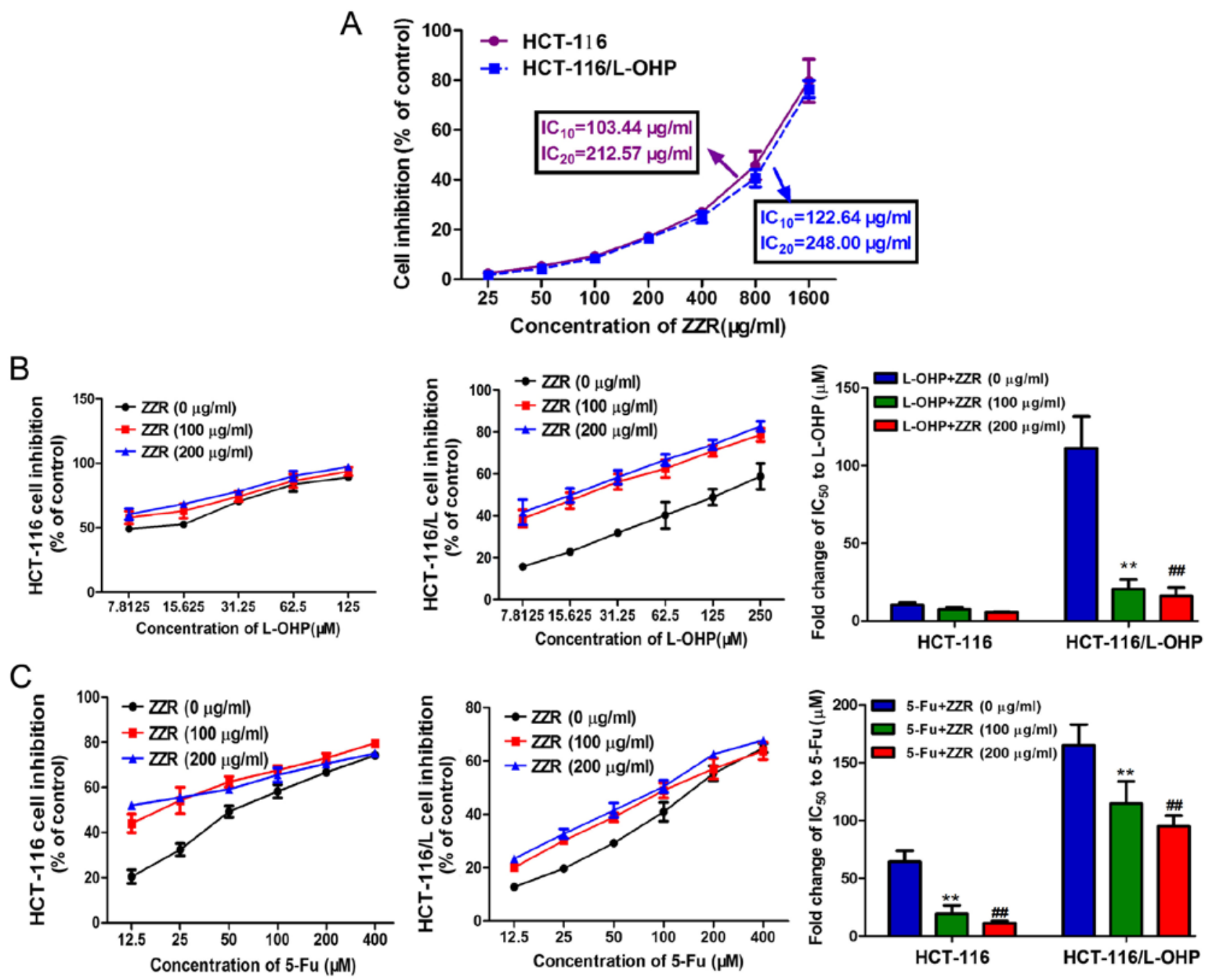

Figure 2. Effect of ZZR on the sensitivity to chemotherapeutic drug in human colorectal cancer cells. (A) Cells were treated with various concentrations of ZZR for $48 \mathrm{~h}$ and analysed by CCK-8. (B) $\mathrm{IC}_{50}$ values of L-OHP on HCT-116 and HCT-116/L-OHP cells exposed to ZZR at 0,100 and $200 \mu \mathrm{g} / \mathrm{ml}$ for $48 \mathrm{~h}$. ${ }^{* *} \mathrm{P}<0.01,{ }^{\# \#} \mathrm{P}<0.01$ vs. ZZR $(0 \mu \mathrm{g} / \mathrm{ml})$. (C) $\mathrm{IC}_{50}$ values of 5-FU on HCT-116 and HCT-116/L-OHP cells exposed to ZZR at $0,100 \mathrm{and} 200 \mu \mathrm{g} / \mathrm{ml}$ for $48 \mathrm{~h}$. ${ }^{* *} \mathrm{P}<0.01,{ }^{\# \#} \mathrm{P}<0.01$ vs. ZZR $(0 \mu \mathrm{g} / \mathrm{ml})$. All data are presented as mean $\pm \mathrm{SD}$ of triplicate independent experiments.

Hedgehog signaling pathway, protein levels of Ptch1, Gli1, and Gli2 were interrogated. As shown in Fig. 4A, the Ptch1, Gli1, and Gli2 protein levels were higher in colorectal cancer MDR cells than that of the sensitive cells. Next, we found ZZR attenuated levels of Gli1 protein in HCT-116/L-OHP cells, without affecting Ptch1 and Gli2 (Fig. 4B). The data suggested that ZZR did not alter the upstream signaling of Hedgehog pathway, such as Ptch1, in HCT-116/L-OHP cells. To determine whether the ZZR modulated Hedgehog pathway activation, we used a specific agonist SAG in the colorectal cancer sensitive cells. As expected, Hedgehog pathway was activated by SAG, but the Gli1 protein level was decreased by ZZR in a dose-dependent manner, suggesting the activation of Hedgehog pathway was dampened by ZZR (Fig. 4C).

ZZR synergizes with chemotherapeutic agents through downregulating Glil in vivo. The in vivo MDR-antagonizing effect of ZZR was evaluated by using colorectal MDR cancer xenograft mice. As shown in Fig. 5A, ZZR and L-OHP combination treatment resulted in a $69.17 \%$ reduction in average tumor volume compared with the controls $\left(1025 \pm 95\right.$ vs. $\left.3352 \pm 285 \mathrm{~mm}^{3}\right)$.
These data indicated that ZZR enhanced the inhibitory effect of L-OHP in vivo. Immunohistochemistry analyses confirmed the lowered levels of Gli1 in tumors treated by ZZR (Fig. 5B). These findings suggested that ZZR was a MDR inhibitor repressing the activation of Hedgehog pathway incolorectal cancer (Fig. 6).

\section{Discussion}

MDR is one of the main factors for colorectal cancer chemotherapy failure (20-22). To overcome MDR, efforts have been made to thwart drug membrane transporters, but broadly applicable inhibitors are to no avail due to severe side effects of the drugs $(23,24)$. In China, Chinese herbal medicines, often prescribed as concoctions, are commonly used as complementary approaches for the prevention and treatment of colorectal cancer (25). Published reports have indicated that adjunctive TCM therapies display remarkable advantages, including maintaining organ functions, improving life quality, ameliorating symptoms, reducing side effects of chemotherapy or radiotherapy, and extending survival time in patients with 
A

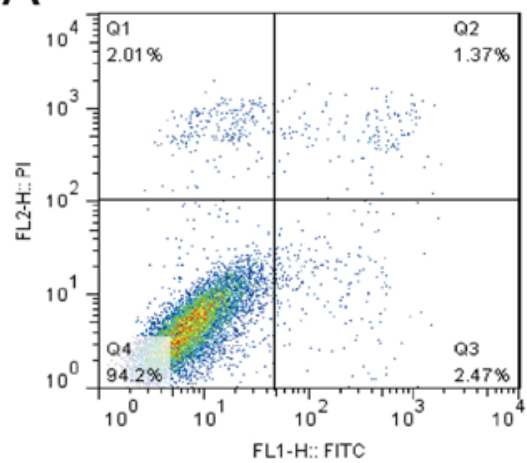

$\mathrm{L}-\mathrm{OHP}+\mathrm{ZZR}(50 \mu \mathrm{g} / \mathrm{ml})$

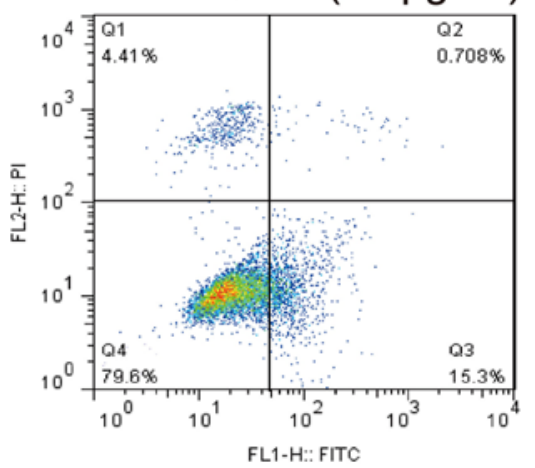

B

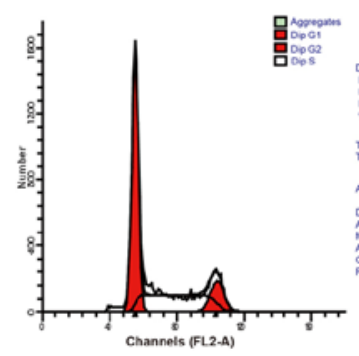

$\mathrm{ZZR}(50 \mu \mathrm{g} / \mathrm{ml})$

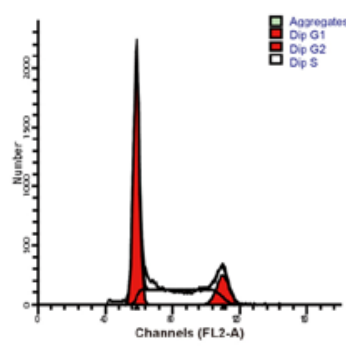

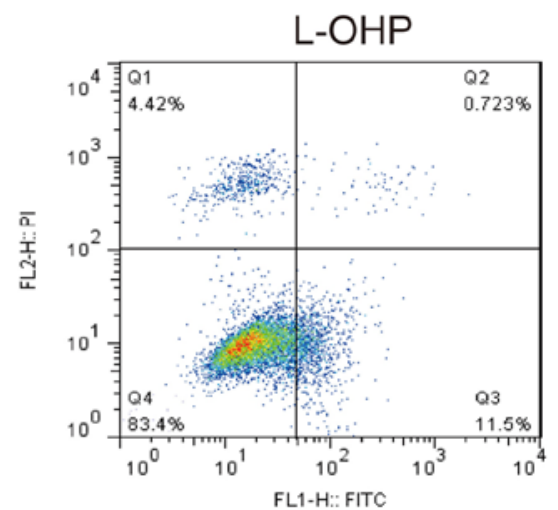

$\mathrm{L}-\mathrm{OHP}+\mathrm{ZZR}(100 \mu \mathrm{g} / \mathrm{ml})$

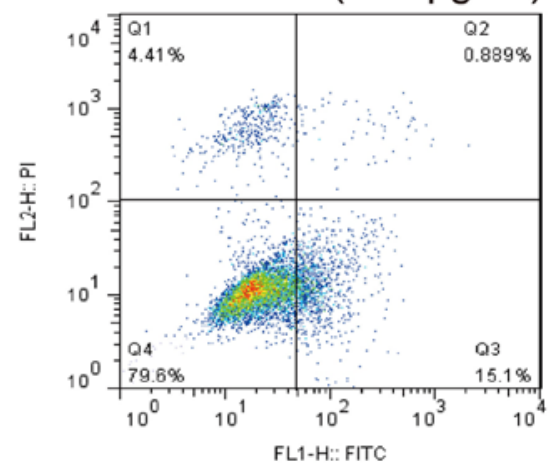

L-OHP

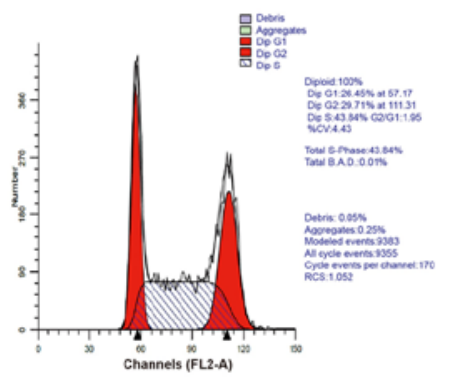

$\mathrm{ZZR}(100 \mu \mathrm{g} / \mathrm{ml})$

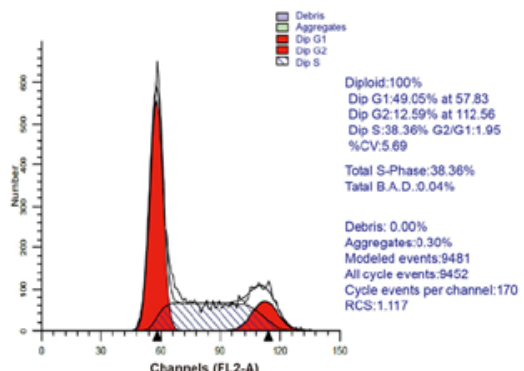

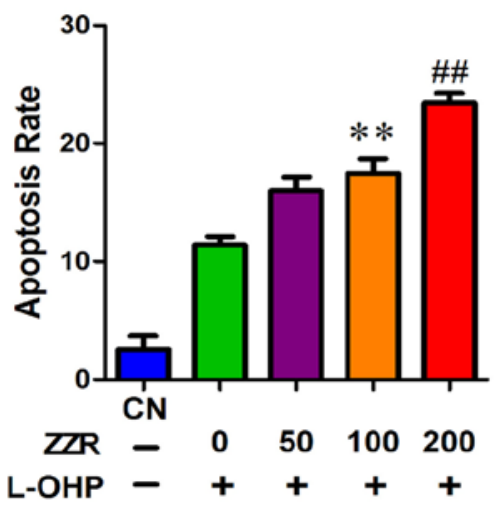

$\mathrm{L}-\mathrm{OHP}+\mathrm{ZZR}(200 \mu \mathrm{g} / \mathrm{ml})$
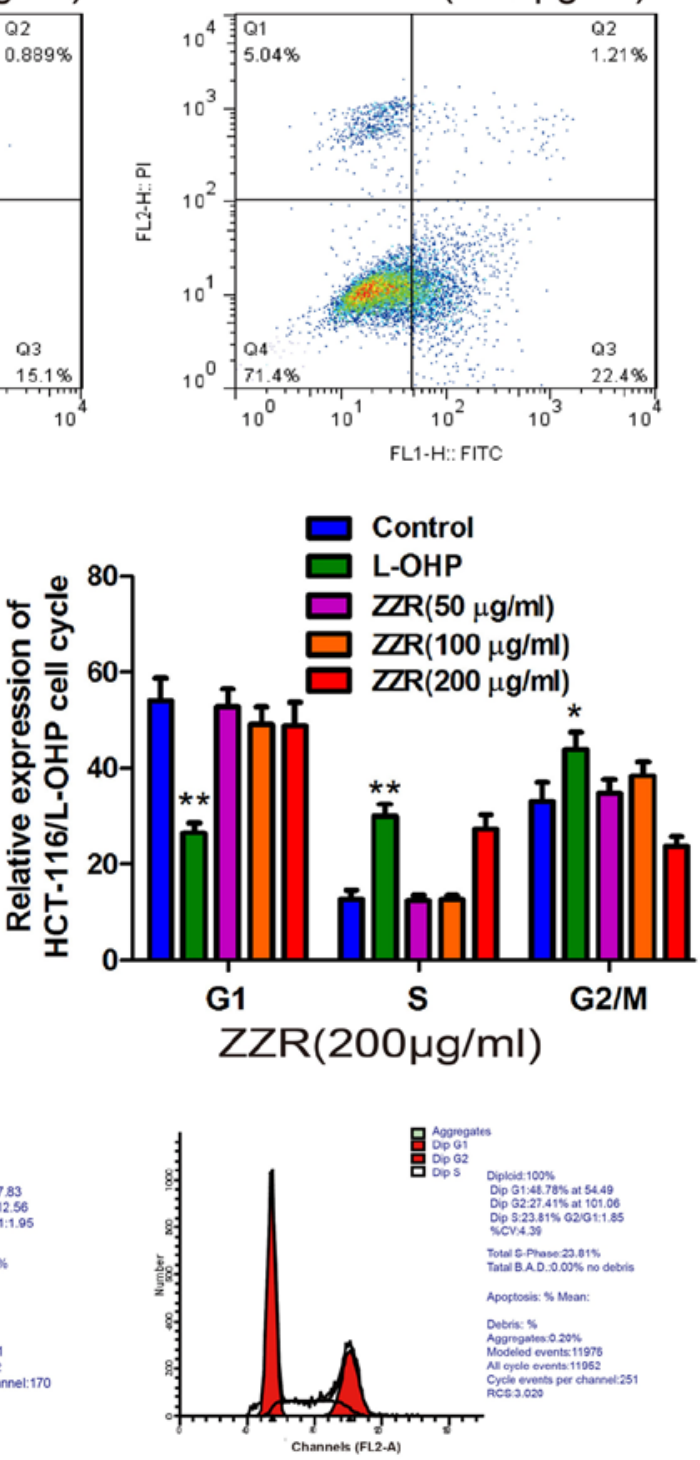

Figure 3. ZZR-induces apoptosis without affecting cell cycle distribution of HCT-116/L-OHP cells. (A) Flow cytometry analysis of cell apoptosis induced by chemotherapeutic agents. Annexin V-FITC/PI binding to HCT-116/L-OHP cells were measured after treatment with L-OHP (10 $\mu \mathrm{M})$ and L-OHP $(10 \mu \mathrm{M})$ combination with ZZR at 50, 100 and $200 \mu \mathrm{g} / \mathrm{ml}$ for $48 \mathrm{~h}$. (B) Flow cytometry analysis was used to distinguish cells in different phases of the cell cycle as HCT-116/L-OHP cells were measured after treatment with L-OHP and ZZR at 50, 100 and $200 \mu \mathrm{g} / \mathrm{ml}$ for $48 \mathrm{~h}$. Data are presented as mean \pm SD of triplicate independent experiments. ${ }^{* *} \mathrm{P}<0.01,{ }^{\# \#} \mathrm{P}<0.01$.

advanced colorectal cancer (26-28). In our previous study, we found that ZZR, a traditional Chinese herb, has significant anticancer effects in enhancing efficacy of chemotherapeutic drugs. Based on this clinical finding, we aimed to explore the
anti-MDR effect of ZZR ethanol extract using in vitro and in vivo models.

In our study, the herbal components of ZZR extract, including Huang-Qi (Radix Astragali), Nv-Zhen-Zi (Fructus 

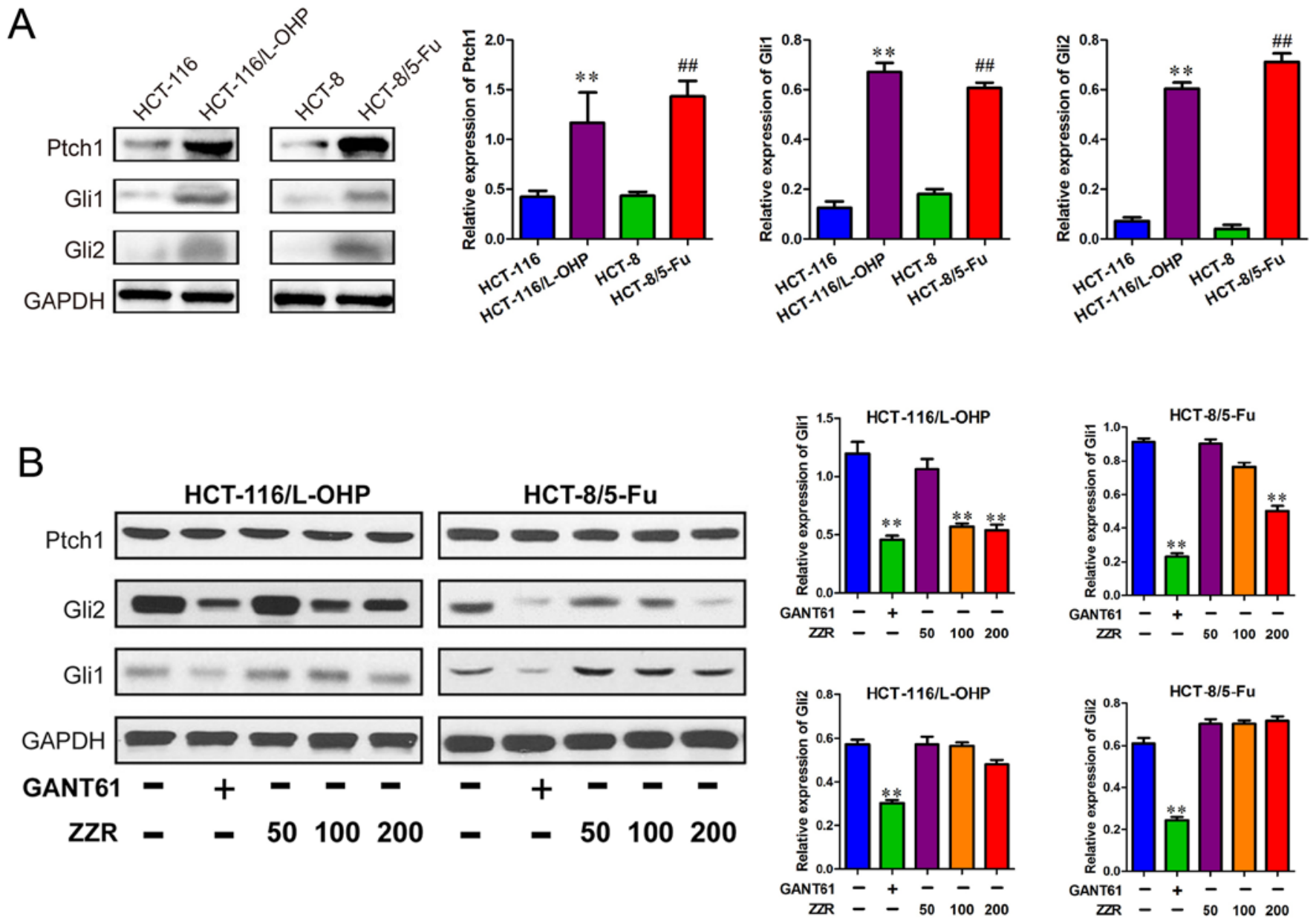

C
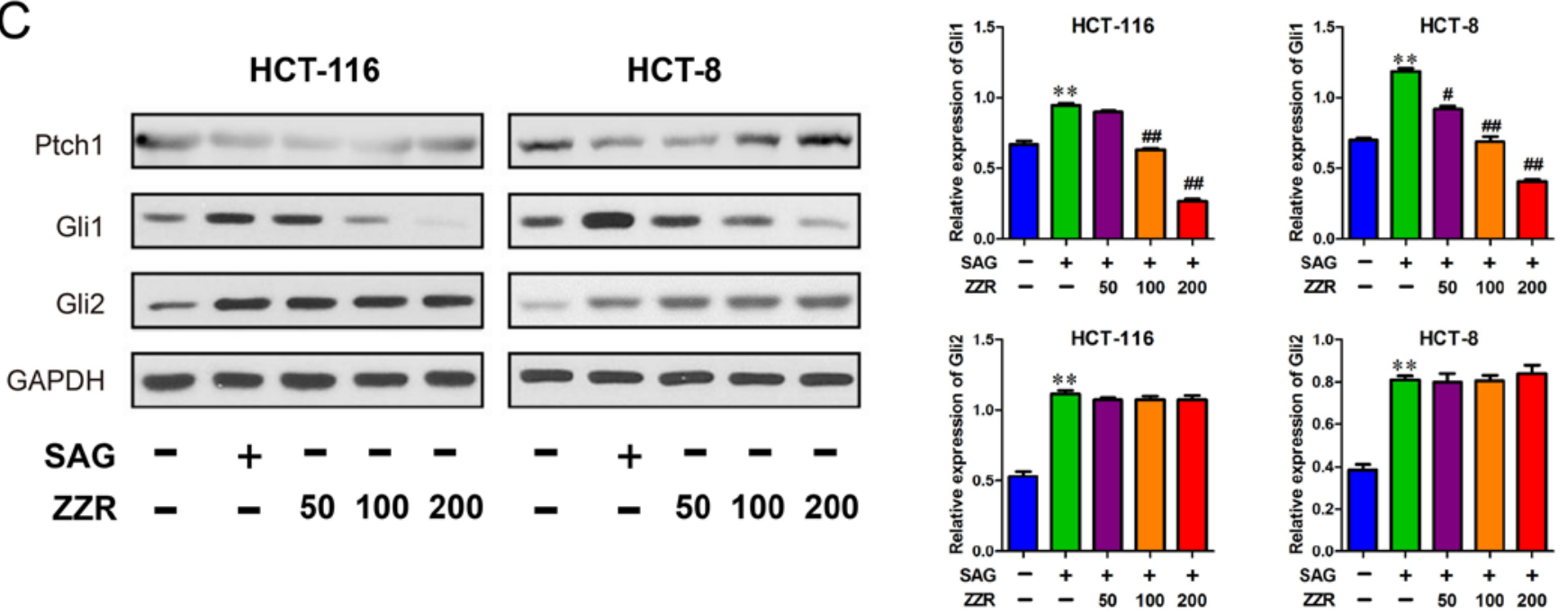

Figure 4. Effects of ZZR on the Hedgehog pathway in sensitive and insensitive colorectal cancer cells. (A) Western blot analysis on the level of Ptch1, Gli1 and Gli2 in sensitive and insensitive colorectal cancer cells. GAPDH was used to ensure equal loading of proteins in each lane. (B) Western blot analysis on the level of Ptch1, Gli1 and Gli2 in colorectal cancer MDR cells treated with ZZR at 50,100 and $200 \mu \mathrm{g} / \mathrm{ml}$ for $48 \mathrm{~h}$, respectively. GAPDH was used to ensure equal loading of proteins in each lane. (C) Western blot analysis on the level of Ptch1, Gli1 and Gli2 in colorectal cancer cells treated with ZZR at 50, 100 and $200 \mu \mathrm{g} / \mathrm{ml}$ for $48 \mathrm{~h}$, respectively. GAPDH was used to ensure equal loading of proteins in each lane. Data are means \pm standard deviation of values from triplicate independent experiments. ${ }^{* *} \mathrm{P}<0.01$ vs. control group. ${ }^{\#} \mathrm{P}<0.05,{ }^{\# \#} \mathrm{P}<0.01$ vs. SAG group,

Ligustri Lucidi), Yi-yi-ren (Semen Coicis), Shi-Jian-Chuan (Salvia Chinensis), Ye-Pu-Tao-Teng (Vitis quinquangularis Rehd), Teng-Li-Gen (Actinidia arguta), and Zhi-Xiang-Fu
(Cyperus rotundus L.), were analyzed by HPLC/ESI-MS. To investigate the anti-MDR effects of ZZR on human colorectal cancer, HCT-116 and its MDR counterpart 
A
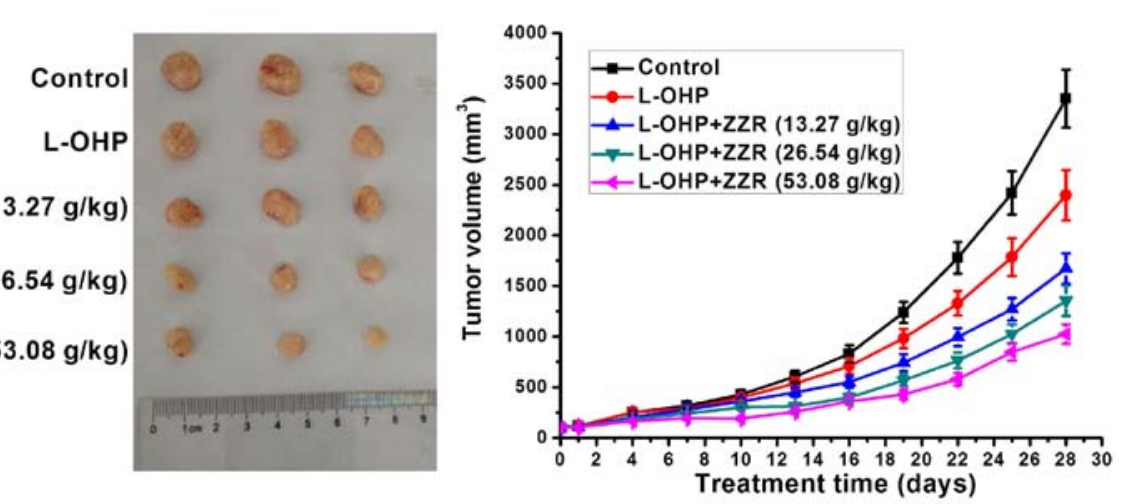

B

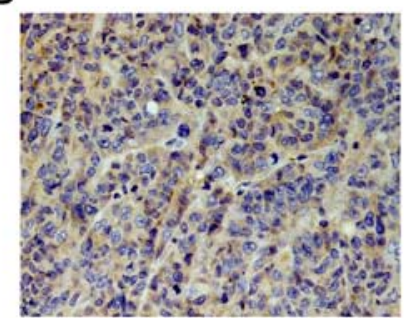

Control

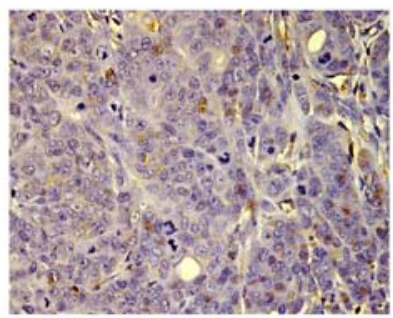

$\mathrm{L}-\mathrm{OHP}+\mathrm{ZZR}(13.27 \mathrm{~g} / \mathrm{kg})$

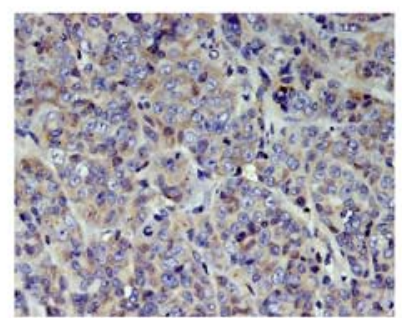

L-OHP

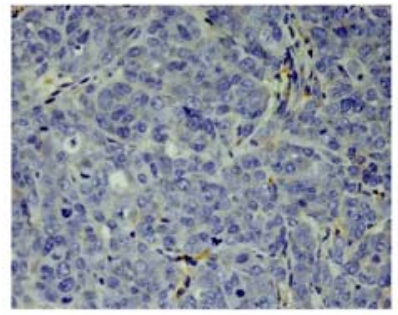

L-OHP+ZZR (26.54g/kg)
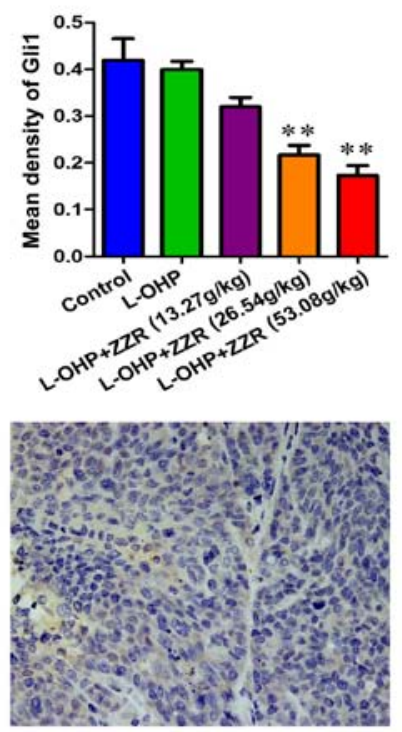

L-OHP+ZZR (53.08g/kg)

Figure 5.ZZR enhances anticancer efficiency of chemotherapeutic reagent in vivo. (A) Tumors removed from nude mice and photographed on the 28th day after administration. Right, tumor volume was quantitated for each sample with ZZR and L-OHP treatment. Data are means \pm SEM. (B) Immunohistochemistry for Gli1 on the subcutaneous transplantation tumor samples in mice as described previously. Magnification, x400. Positive cells were stained brown.

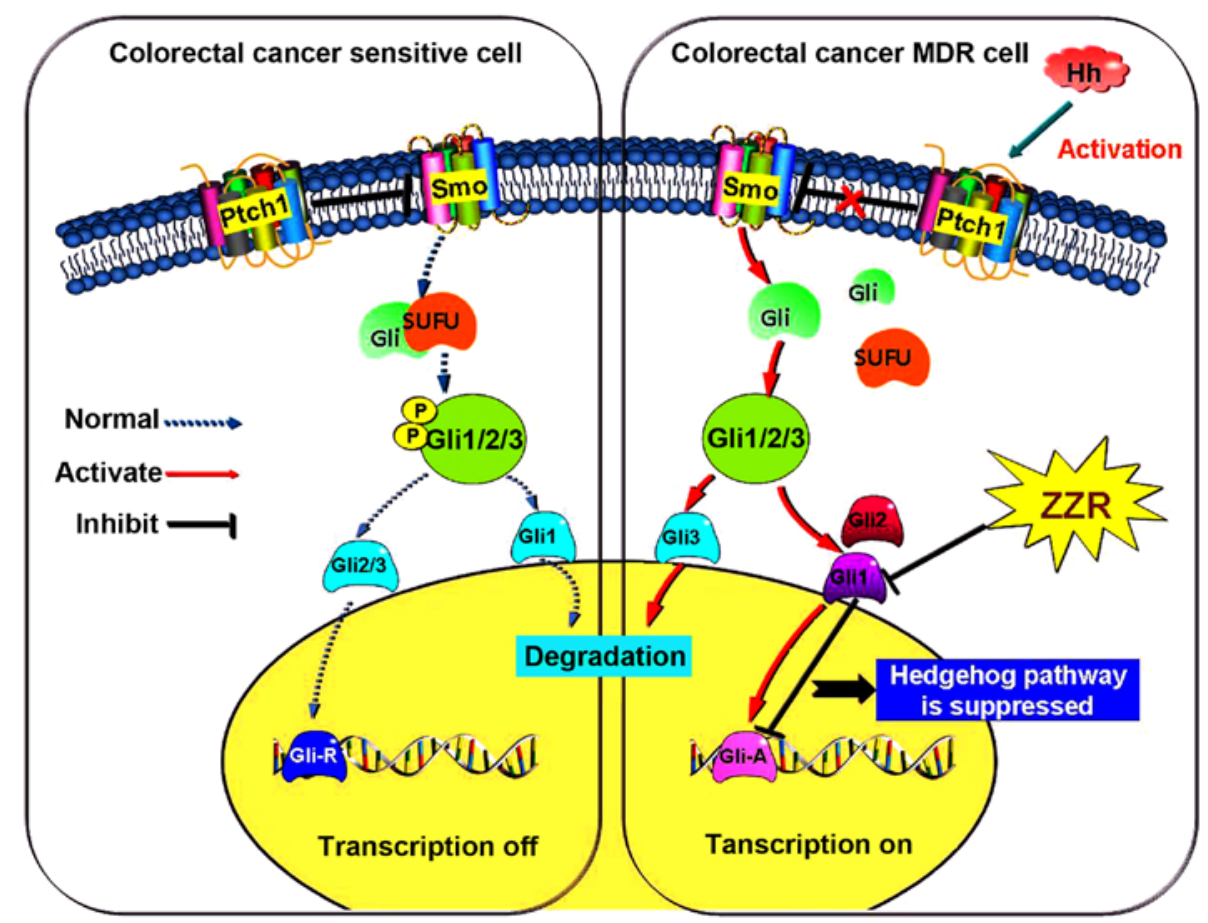

Figure 6. Proposed working model of ZZR on the Hedgehog signaling pathway-mediated MDR in colorectal cancer cells. 
HCT-116/L-OHP cells were treated with ZZR, and the nontoxic dose of ZZR was determined. The result suggested that concentrations of ZZR $<200 \mu \mathrm{g} / \mathrm{ml}$ are safe in experiments in vitro. Furthermore, cell viability assays were performed in HCT-116 and HCT-116/L-OHP cells to determine their relative sensitivity in increasing concentrations of chemotherapy drugs, L-OHP and 5-FU. Data showed that HCT-116/L-OHP cells are much more sensitive to death induced by chemotherapeutics after ZZR treament, when compared with its parental cells.

In our study, we found ZZR synergized with chemotherapeutic drugs to enhance cell apoptosis in a time-dependent manner, consistent with what several previous reports have shown with traditional Chinese prescriptions and formulae $(29,30)$. However, in our study with ZZR, we did not observe any disturbance on cell cycle. One possible explanation is because of the interaction between different herb constituents of this formula which could lead to complicated effects. These findings are in accordance with several reports showing opposite effects for some antitumor drugs, causing apoptosis but not cell cycle alterations (17,31).

Earlier study from our laboratory revealed an association between Hedgehog pathway and P-gp in MDR colorectal cancer cells (32). Here we extended that study by using another two MDR colorectal cancer cell lines, and found ZZR perturbed the expression levels of Ptch1, Gli1 and Gli2. We uncovered that expression levels of Ptch1, Gli1 and Gli2 were higher in resistant HCT-116/L-OHP and HCT-8/5-FU cells than that of their parental sensitive cells. It was reported that Hedgehog signaling pathway was activated in MDR cancer cells (33). In this study, in HCT-116/L-OHP and HCT-8/5-FU cells, we observed elevated protein levels of Ptch1, Gli1, and Gli2, among which ZZR treatment specifically repressed the levels of Gli1.

We next investigated the in vivo therapeutic potential of ZZR. Indeed, animal experiment results showed a smilar synergistic anticancer effect of ZZR with chemotherapy drugs on resistant colorectal cancer cell xenografts. Our data further confirmed that ZZR markedly inhibited Glil protein level in the xenograft tumors of MDR colorectal cancer cells.

In conclusion, the present study for the first time demonstrated that ZZR suppresssed Gli1 protein levels in colorectal cancer through inhibiting Hedgehog signaling pathway. The traditional Chinese prescription and formula ZZR, used alone or incombination with chemotherapeutic agents, may provide promising approach for the treatment of drug-resistant colorectal cancer.

\section{Acknowledgements}

This research was supported by the project from Shanghai Municipal Commission of Health and Family Planning (no. ZY3CCCX11009), the National Natural Science Foundation of China (nos. 81373862, 81403360 and 81573805), and Program from Xinjiang Autonomous Region (no. 2013911125).

\section{References}

1. Sui H, Cai GX, Pan SF, Deng WL, Wang YW, Chen ZS, Cai SJ, Zhu HR and Li Q: miR200c attenuates P-gp-mediated MDR and metastasis by targeting JNK2/c-Jun signaling pathway in colorectal cancer. Mol Cancer Ther 13: 3137-3151, 2014.
2. Sui H, Zhu L, Deng W and Li Q: Epithelial-mesenchymal transition and drug resistance: Role, molecular mechanisms, and therapeutic strategies. Oncol Res Treat 37: 584-589, 2014.

3. Zhang YK, Zhang GN, Wang YJ, Patel BA, Talele TT, Yang DH and Chen ZS: Bafetinib (INNO-406) reverses multidrug resistance by inhibiting the efflux function of ABCB1 and ABCG2 transporters. Sci Rep 6: 25694, 2016.

4. Nüsslein-Volhard C and Wieschaus E: Mutations affecting segment number and polarity in Drosophila. Nature 287: 795-801, 1980.

5. Lee RT, Zhao Z and Ingham PW: Hedgehog signalling. Development 143: 367-372, 2016.

6. Flemban A and Qualtrough D: The potential role of Hedgehog signaling in the luminal/basal phenotype of breast epithelia and in breast cancer invasion and metastasis. Cancers (Basel) 7: 1863-1884, 2015.

7. Laukkanen MO and Castellone MD: Hijacking the Hedgehog pathway in cancer therapy. Anticancer Agents Med Chem 16: 309-317, 2016.

8. Kling JC and Blumenthal A: Roles of WNT, NOTCH, and Hedgehog signaling in the differentiation and function of innate and innate-like lymphocytes. J Leukoc Biol jlb.1MR0616-272R, 2017.

9. Xie J, Bartels CM, Barton SW and Gu D: Targeting Hedgehog signaling in cancer: Research and clinical developments. Onco Targets Ther 6: 1425-1435, 2013.

10. Lee MY, Sun L and Veltmaat JM: Hedgehog and Gli signaling in embryonic mammary gland development. J Mammary Gland Biol Neoplasia 18: 133-138, 2013.

11. Shevde LA and Samant RS: Nonclassical Hedgehog-GLI signaling and its clinical implications. Int J Cancer 135: 1-6, 2014.

12. Malhotra GK, Zhao X, Band $\mathrm{H}$ and Band V: Shared signaling pathways in normal and breast cancer stem cells. J Carcinog 10: 38, 2011.

13. Li WY, Chan SW, Guo DJ, Chung MK, Leung TY and Yu PH: Water extract of Rheum officinale Baill. induces apoptosis in human lung adenocarcinoma A549 and human breast cancer MCF-7 cell lines. J Ethnopharmacol 124: 251-256, 2009.

14. Rasul A, Yu B, Yang LF, Ali M, Khan M, Ma T and Yang H: Induction of mitochondria-mediated apoptosis in human gastric adenocarcinoma SGC-7901 cells by kuraridin and Nor-kurarinone isolated from Sophora flavescens. Asian Pac J Cancer Prev 12: 2499-2504, 2011.

15. Sui H, Zhu HR, Wu J, Nikitin AY, Cai JF, Fan ZZ and Li Q: Effects of Jianpi Jiedu Recipe on reversion of P-glycoproteinmediated multidrug resistance through COX-2 pathway in colorectal cancer. Chin J Integr Med 20: 610-617, 2014.

16. Sun B, Chen Y, Xiang T, Zhang L, Chen Z, Zhang S, Zhou H and Chen S: The Chinese herb Jianpijiedu contributes to the regulation of OATP1B2 and $\mathrm{ABCC} 2$ in a rat model of orthotopic transplantation liver cancer pretreated with food restriction and diarrhea. BioMed Res Int 2015: 752850, 2015.

17. Sui H, Liu X, Jin BH, Pan SF, Zhou LH, Yu NA, Wu J, Cai JF, Fan ZZ, Zhu HR, et al: Zuo Jin Wan, a traditional Chinese herbal formula, reverses P-gp-mediated MDR in vitro and in vivo. Evid Based Complement Alternat Med 2013: 957078, 2013.

18. Sui H, Xu H, Ji Q, Liu X, Zhou L, Song H, Zhou X, Xu Y, Chen Z, Cai J, et al: 5-hydroxytryptamine receptor (5-HT1DR) promotes colorectal cancer metastasis by regulating Axin1/ $\beta$-catenin/ MMP-7 signaling pathway. Oncotarget 6: 25975-25987, 2015.

19. Kolinsky K, Shen BQ, Zhang YE, Kohles J, Dugan U, Zioncheck TF, Heimbrook D, Packman K and Higgins B: In vivo activity of novel capecitabine regimens alone and with bevacizumab and oxaliplatin in colorectal cancer xenograft models. Mol Cancer Ther 8: 75-82, 2009.

20. Martins A, Sipos P, Dér K, Csábi J, Miklos W, Berger W, Zalatnai A, Amaral L, Molnár J, Szabó-Révész P, et al: Ecdysteroids sensitize MDR and non-MDR cancer cell lines to doxorubicin, paclitaxel, and vincristine but tend to protect them from cisplatin. BioMed Res Int 2015: 895360, 2015.

21. Wang S, Wang L, Shi Z, Zhong Z, Chen M and Wang Y: Evodiamine synergizes with doxorubicin in the treatment of chemoresistant human breast cancer without inhibiting P-glycoprotein. PLoS One 9: e97512, 2014.

22. Wang Z, Sun X, Feng Y, Liu X, Zhou L, Sui H, Ji Q, e Q, Chen J, Wu L, et al: Dihydromyricetin reverses MRP2-mediated MDR and enhances anticancer activity induced by oxaliplatin in colorectal cancer cells. Anticancer Drugs 28: 281-288, 2017.

23. Sharom FJ: ABC multidrug transporters: Structure, function and role in chemoresistance. Pharmacogenomics 9: 105-127, 2008. 
24. Wilkens S: Structure and mechanism of ABC transporters. F1000Prime Rep 7: 14, 2015.

25. Deng W, Sui H, Wang Q, He N, Duan C, Han L, Li Q, Lu M and Lv S: A Chinese herbal formula, Yi-Qi-Fu-Sheng, inhibits migration/invasion of colorectal cancer by down-regulating MMP-2/9 via inhibiting the activation of ERK/MAPK signaling pathways. BMC Complement Altern Med 13: 65, 2013.

26. Cao P, Selvan SR, Küpeli Akkol E, Wang N, Yang H and Cheng X: Complementary and alternative medicine in cancer prevention and therapy. Evid Based Complement Alternat Med 2015: 639372, 2015.

27. Yao CJ, Chow JM, Yang CM, Kuo HC, Chang CL, Lee HL, Lai IC, Chuang SE and Lai GM: Chinese herbal mixture, Tien-Hsien liquid, induces $\mathrm{G} 2 / \mathrm{M}$ cycle arrest and radiosensitivity in MCF-7 human breast cancer cells through mechanisms involving DNMT1 and Rad51 downregulation. Evid Based Complement Alternat Med 2016: 3251046, 2016.

28. Chen T, Wang Q, Li Y, Huang H and Hu W: Chinese herbal formula QHF inhibits liver cancer cell invasion and migration. Exp Ther Med 11: 2413-2419, 2016.

29. Li J, Sun GZ, Lin HS, Pei YX, Qi X, An C, Yu J and Hua BJ: The herb medicine formula 'Yang Wei Kang Liu' improves the survival of late stage gastric cancer patients and induces the apoptosis of human gastric cancer cell line through Fas/Fas ligand and Bax/Bcl-2 pathways. Int Immunopharmacol 8: 1196-1206, 2008 .
30. Wu G, Chu J, Huang Z, Ye J, Chen P, Zheng C, Li X, Liu X and Wu M: Xiao Jin Wan, a traditional Chinese herbal formula, inhibits proliferation via arresting cell cycle progression at the $\mathrm{G} 2 / \mathrm{M}$ phase and promoting apoptosis via activating the mitochondrial-dependent pathway in U-2OS human osteosarcoma cells. Int J Oncol 42: 1070-1080, 2013.

31. Sui H, Pan SF, Feng Y, Jin BH, Liu X, Zhou LH, Hou FG, Wang WH, Fu XL, Han ZF, et al: Zuo Jin Wan reverses P-gp-mediated drug-resistance by inhibiting activation of the PI3K/Akt/NF- $\mathrm{KB}$ pathway. BMC Complement Altern Med 14: 279, 2014

32. Liang W, Wang H, Tang Y, Cai SB, Zhang X and Wang SP: The effect and mechanism of Zhizhen Recipe on multidrug resistance in human colorectal cancer cell lines through Hedgehog signaling pathway. Chin J Integr Trad W Med Dig 23: 151-155, 2015 (In Chinese)

33. Zhang XL, Shi HJ, Wang JP, Tang HS and Cui SZ: MiR-218 inhibits multidrug resistance (MDR) of gastric cancer cells by targeting Hedgehog/smoothened. Int J Clin Exp Pathol 8: 6397-6406, 2015. 